\title{
THE PHASE DIAGRAM FOR THE SINE-GORDON MODEL WITH TWO UMKLAPP TERMS
}

\author{
A. Bjeliš and M. Latković \\ Department of Theoretical Physics, Faculty of Sciences \\ University of Zagreb, P.O.B. 162, 41001 Zagreb, Croatia
}

\begin{abstract}
We study the Landau free energy for a uniaxial ordering, taking into account two Umklapp terms of comparable strengths (those of the third and fourth order). Exploring the analogy with the well-known nonintegrable classical mechanical problem of two mixed nonlinear resonances, we complete the previous studies of the corresponding phase portrait by calculating numerically periodic solutions, including those far from the separatrices. It is shown that in the physical range of parameters only periodic configurations are absolutely stable. We determine for the first time the complete thermodynamic phase diagram and show that, in contrast to some earlier claims, the wave number of the ordering does not pass through the devil's staircase, but through a finite number of steps which decreases as the amplitudes of the Umklapp terms increase.
\end{abstract}

05.70.-a, 64.70.Rh

Typeset using REVTEX 
The sine-Gordon model [1,2] which is widely used in the studies of the uniaxial incommensurate-commensurate (IC) transitions [3], is based on a few intricate approximations. By one of them only one, presumably dominant, phase dependent Umklapp term is retained in the Landau expansion of the free energy. In this Letter we critically examine this approximation by including two Umklapp terms which favor different commensurabilities, i.e. those of the third and fourth order. Like in other extensions of the sine-Gordon model [4], one encounters a nonintegrable problem, well-known in the modern classical mechanics [9, 10]. As a consequence, the thermodynamic phase diagram for the IC transition qualitatively differs from that of the simple sine-Gordon model.

It is well-known that continuous nonintegrable models [7, \&] and discrete models [4 6] for the IC transition lead to similar descriptions of uniaxial orderings. The analyses were however usually made for limited ranges of values of physical parameters which enter into models. Also, the continuous models were examined only in the parts of phase space close to separatrices, characterized by dilute soliton lattices. With such restrictions it was not possible to determine complete thermodynamic phase diagrams which are the basis for any discussion of particular real systems. In this Letter we combine two numerical methods, with the aim to investigate the entire phase space and to find the regions of absolute stability for particular solutions in the plane spanned by two control parameters (out of three present in the model). It will be shown that, due to the gradual disappearance of quasiperiodic solutions and the formation of chaotic layers, the phase diagram is (almost) entirely covered by periodic configurations.

The model is defined by the free energy functional

$$
F=\int d x\left[\frac{1}{2}\left(\frac{d \phi}{d x}\right)^{2}+B \cos \left(3 \phi+3\left(\frac{\pi}{6}-\delta_{4}\right) x\right)+C \cos \left(4 \phi-4 \delta_{4} x\right)\right]
$$

with $\phi$ being the phase of the order parameter. $\delta_{4} \equiv Q-Q_{4}$, where $Q$ is the wave number of ordering in the absence of Umklapp terms and $Q_{4}=2 \pi / 4$ is the commensurate wave number of the fourth order. (We put everywhere the lattice constant equal to unity.) By assumption $Q$ is placed somewhere between $Q_{4}$ and the commensurate wave number of the 
third order $Q_{3}=2 \pi / 3$. In that case the Umklapp terms of third and fourth order which are included into the Landau expansion (1) might be of comparable strengths. E. $g$. the Umklapp term of $m$-th order for charge and spin density waves is proportional to the $m$-th power of the amplitude of the order parameter [11]. Thus, at temperatures not far below the critical temperature $T_{c}$ of the incommensurate ordering, the third order Umklapp term should not be omitted due to the dominance of the coupling constant $B$ with respect to $C$. On the other hand, for small values of $\delta_{4}\left(e \cdot g\right.$. for $\left.\delta_{4} \ll \pi / 6-\delta_{4}\right)$ the closeness of the wave number to the commensurate point $Q_{4}$ favors the fourth order Umklapp term. The similar arguments can be invoked for other types of uniaxial orderings. Note that the example (1) is the most interesting one since it includes two Umklapp terms which are of the lowest possible orders in the amplitude of the order parameter. (The model for the commensurate lock-in at $Q=\pi$ is of essentially different type 12,13 ).

The competition between two periodicities in the expansion (1) takes place in the nonlinear terms which are formed on the purely symmetry grounds. It is present in both the $\phi$ dependence and the "nonautonomous" $x$-dependence of these terms. We recall that the starting functional of Fradkin et al [7], which is appropriate for the approximate treatment of the ordering in the presence of mobile defects [B], contains only the competition of the latter type.

The extremalization of the functional (目) leads to the Euler-Lagrange (EL) equation

$$
\phi^{\prime \prime}+3 B \sin \left[3 \phi+3\left(\pi / 6-\delta_{4}\right) x\right]+4 C \sin \left(4 \phi-4 \delta_{4} x\right)=0 .
$$

Among all solutions of this equation we are looking for that which is the absolute minimum of the mean free energy density in the eq. (1) [14]. To this end we develop a procedure which is complementary to those usually performed in the classical mechanical analyses of the phase portrait of eq. (2) (see e. g. refs. [9,[10]). Standard methods of direct integration of eq. (2), which were widely used in the calculations of quasiperiodic and chaotic orbits, cannot be extended to the isolated periodic solutions, which are orbitally unstable in the most interesting cases of phase portraits with finite chaotic layers. However, it appears that 
just these solutions have the main role in the thermodynamic phase diagram. We therefore calculate them separately, by treating eq. (2) as the boundary value problem. Combining these two methods we can follow all relevant trajectories in the phase portrait and extract the trajectory for which the functional (11) has the absolute minimum.

The functional (11) and the EL equation (21) do not possess the continuous translational symmetry in $x$, but are invariant under the combinations of discrete translations in both $x$ and $\phi$ [15. All solutions of eq. (21) can be thus grouped into degenerate subsets of solutions connected by these translations. In the further considerations of the periodic solutions we enumerate these subsets and represent each subset by one solution.

It is easy to determine possible periods of the periodic solutions, defined by

$$
\phi(x+P)=\phi(x)+\phi_{P} .
$$

Inserting eq. (3) into the EL equation (2), one gets the necessary conditions

$$
P=4 k+3 l, \quad \phi_{P}=\delta_{4} P-l \pi / 2,
$$

where $k$ and $l$ are integers. The values of $P$ are $\phi_{P}$ are thus discretized (in contrast to the simple sine-Gordon case) and denote allowed intervals of variables $x$ and $\phi$ in the boundary value problem. Note that the solutions with finite values of $\phi_{P}$ are rotational (i.e. have finite winding numbers).

The boundary conditions for the periodic solutions with the values of $P$ and $\phi_{P}$ given by eq. (田) are defined by $\phi_{k l}\left(x=x_{i}\right), \phi_{k l}\left(x=x_{f}=x_{i}+P\right)=\phi_{k l}\left(x=x_{i}\right)+\phi_{P}$ and $\phi_{k l}^{\prime}\left(x=x_{i}\right)=\phi_{k l}^{\prime}\left(x=x_{f}\right)$. Using two different numerical methods [16] we undertake a systematic search of such conditions for periods $P$ up to $\approx 100$. Both numerical procedures give at most two solutions with the same values of $k$ and $l$, defined by two isolated points in the two-dimensional plane of boundary conditions $\left[\phi_{k l}\left(x=x_{i}\right), \phi_{k l}^{\prime}\left(x=x_{i}\right)\right]$ [17. We noticed [15] that these points sometimes have jumps and discontinuities as the functions of the parameters $B$ and $C$. In particular, a given periodic solution may cease to exist as the nonlinearity strengthens, the general tendency being that the solutions with smaller periodicities are more robust as $B$ and/or $C$ increase. 
When both exist, two solutions with same values of $P$ and $\phi_{P}$ have different values of the free energy (四). Both have odd symmetry [the only possible for rotational solutions], but with different positions of inversion points $\left[x_{0}, \phi\left(x_{0}\right)\right]$. It easily follows from the differential equation (2) that $x_{0}$ necesarily has only integer and half-integer values, while $\phi\left(x_{0}\right)=$ $x_{0} \delta_{4}-m \pi / 4, \quad m=0, \pm 1, \pm 2, \ldots$. This is indeed the case with all our numerical solutions. With the odd symmetry taken into account, the numerical procedure is further simplified, since it remains to determine only one continuous boundary condition $\left[\phi_{k l}^{\prime}\left(x=x_{0}\right)=\phi_{k l}^{\prime}(x=\right.$ $\left.\left.x_{0}+P\right)\right]$ and two discrete numbers $\left[x_{0}\right.$ and $\left.\phi\left(x_{0}\right)\right]$ per each solution. This analysis 15 shows that the particular values of $x_{0}$ and $\phi\left(x_{0}\right)$ for a given solution are related to the evenness and oddness of the corresponding integers $k$ and $l$. Further below we follow only periodic solutions with the lower free energy (1), and characterize each such solution by a pair of integers $(k, l)$.

The structure factor for a given periodic solution $(k, l)$ has equidistant satellites, whose positions with respect to the the wave number $Q$ are given by

$$
q_{n} \equiv \frac{\phi_{P}}{P}+\frac{2 n \pi}{P}=\delta_{4}+\pi \frac{2 n-l / 2}{4 k+3 l}, \quad n=0, \pm 1, \pm 2, \ldots
$$

The position of the fundamental satellite $q_{0}$ is determined by the total slope of the function $\phi_{k l}(x)$, while the higher harmonics $q_{n}, n \neq 0$ appear due to its periodic modulation.

In the next step we determine mean free energies of particular solutions. For illustration, we show in Figs.1a-c mean free energies of periodic, quasi-periodic and chaotic solutions with one fixed $\left[\phi\left(x_{i}=3 / 2\right)=0\right]$ and one variable $\left[\phi_{0}^{\prime} \equiv \phi^{\prime}\left(x_{i}=3 / 2\right)\right]$ initial condition, choosing $\delta_{4}=\pi / 12$ and three representative values of $B$ and $C$. For all periodic solutions shown in these figures $x_{i}=3 / 2$ coincides with the point of odd symmetry $x_{0}$. Since these figures remain qualitatively same for other choices of the "initial" point $x_{i}$, they enable some general conclusions on the relationship between free energies of various types of solutions.

The situation in which the parts of the phase space with the rotational flow are still free of chaos is presented in Fig.1a. It is evident that there is a periodic solution which has lower mean free energy than all other calculated periodic and quasiperiodic solutions. 
The same numerical conclusion is obtained for other finite values of $B$ and $C$. It should be however mentioned that the comparison of mean free energies of periodic and quasiperiodic configurations becomes more and more subtle as $B$ and/or $C$ decrease, so that in this limit one cannot rigorously exclude the absolute thermodynamic stability of some quasiperiodic solution [5 [7].

We turn from this intricate and numerically unpleasant limit to the intermediate values of $B$ and/or $C$ for which both, quasiperiodic and chaotic, layers are present in the rotational part of the phase space [Fig.1b], and finally to the regime [9]

$$
\sqrt{B}+\sqrt{C}>\pi / 12
$$

in which there is no quasiperiodic solution and this part is covered by a single chaotic layer (Fig.1c). [Note that the wings on the left and right sides in Figs.1.a-c come from the thermodynamically uninteresting oscillating solutions of eq. (2).] Since the numerical integration of eq. (2) cannot extract a single chaotic solution (due to its orbital instability), the plateaux in Figs.1b,c strictly represent mean free energies within a given chaotic layer, i.e. each plateau represents one isolated chaotic layer in the phase space. Still, it is plausibly expected [7] that each particular chaotic solution fully diffuses inside "its" layer, so that its mean free energy coincides with one of the plateaux in Fig.1. Periodic solutions are immersed into these chaotic layers as isolated points. Their free energies are discontinuous (needle-like) minima well below all chaotic plateaux [see Figs.1b (inset) and 1c]. As it was already noticed, the number of surviving periodic solutions decreases with the increase of "chaoticity" of the phase space, measured by the left-hand side of the inequality (6).

The numerical results illustrated by Figs.1a-c are the basis for the construction of the thermodynamic phase diagram in the three-dimensional space of parameters $B, C$ and $\delta_{4}$. One possible visualization is presented in Figs.2 and 3 in which we fix $B$, vary $C$ and $\delta_{4}$ [18], and follow the fundamental wave number $q_{0}$ [eq. (5)] and the free energy of the

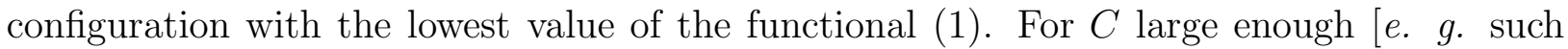
that the inequality (6) is satisfied], the system passes through small number of periodic 
configurations as $\delta_{4}$ varies. Other existing periodic solutions have free energies which are larger and increase as the period $P$ increases [15]. Since it does not seem probable that the further solutions, which have even higher periods and were not calculated numerically, would not follow this tendency, we conclude that there are no other solutions which would fill the phase diagram between the neighboring plateaux shown in the Fig.2.

As $C$ decreases the number of periodic configurations increases through a series of bifurcations, while the dependences of the free energy and the wave number on $\delta_{4}$, although discontinuous, approach that of the simple sine-Gordon limit $C=0$. The number of absolutely stable periodic configurations still remains finite (and smaller than the number of numerically calculated periodic solutions), even for $C \approx 0.001-0.002$ when there are no chaotic layers in the rotational part of phase space (except those in the regions of separatrices) (Fig.1a). In other words, our numerical results suggest that the staircase from Fig.2 remains "harmless" [19] (i.e. not fractal) as long as $C$ is finite (or at least not too small). The harmless staircase with first order transition between neighboring periodic solutions is expected to be closely connected with the participation of dense soliton lattices in our phase diagram. Then it is inappropriate to use the picture of well separated solitons with a repulsive effective interaction, which was usually the basis for the conclusions that the wave number $q_{0}$ may pass through the complete or incomplete devil's staircase [6, [].

In conclusion, we stress that the periodic solutions are of the central importance for the thermodynamics of systems modelled by the free energy (11). In the physical range of parameters ( $B$ and $C$ finite), only these solutions (i.e. the commensurate configurations) have the property of absolute thermodynamic stability. In the phase space they are isolated and immersed in a mostly chaotic environment, whose mean free energies represent characteristic energetic barriers which the system has to overcome in order to replace one periodicity by another. Such barriers might be intrinsic sources [20] of memory effects and thermal hystereses which are frequently observed in uniaxial systems with a variable wave number of ordering [3]. We recall that the thermodynamic significance of periodic and chaotic solutions is just opposite to that of the classical mechanical counterpart of the model (1). Mechanical 
periodic trajectories cannot be realized due to their orbital instability, so that the rotational motion of a strongly nonintegrable classical mechanical system is entirely chaotic. 


\section{REFERENCES}

[1] W. L. McMillan, Phys. Rev. B 14, 1496 (1976).

[2] L. N. Bulaevski and D. I. Khomski, Zh. Eksp. Teor. Fiz. 74, 1863 (1978) [Sov. Phys. JETP 74, 971 (1978)].

[3] See, e.g., H. Z. Cummins, Phys. Rep. 185, 211 (1990).

[4] S. Aubry, J. Physique 44, 147 (1983), and in Statics and dynamics of nonlinear systems, edited by G. Benedek, H. Bilz and R. Zeyher (Springer-Verlag, Berlin, 1983), p.126.

[5] P. Bak, Rep. Prog. Phys. 45, 587 (1982).

[6] B. Joos, B. Bergersen, R. J. Gooding and M. Plischke, Phys. Rev. B 27, 467 (1983).

[7] E. Fradkin, O. Hernandez, B. A. Huberman and R. Pandit, Nucl. Phys. B 215, 137 (1983).

[8] G. Errandonea, Phys. Rev. B 33, 6261 (1986).

[9] B. V. Chirikov, Phys. Rep. 52, 263 (1979).

[10] D. F. Escande, Phys. Rep. 121, 165 (1985).

[11] P. A. Lee, T. M. Rice and P. W. Anderson, Solid State Commun.14, 703 (1974).

[12] A. Michelson, Phys. Rev. B 16, 577 (1977).

[13] V. Dananić, A. Bjeliš, M. Rogina and E. Coffou, Phys. Rev. A 46, 3551 (1992); V. Dananić and A. Bjeliš, Phys. Rev. E 50, 3900 (1994).

[14] In the present work we shall not consider other (metastable) solutions which minimize the free energy (1), i.e. we do not determine ranges of coexistence of two or more configurations, but only the first-order transition lines.

[15] M. Latković and A. Bjeliš, to be published. 
[16] Generally we used the finite-difference method with deferred correction technique [see The NAG Fortran Library Manual, The Numerical Algorithms Group Limited, 1991]. The faster Runge-Kutta-Merson integration method with the Newton shooting technique was used as an auxiliary method whenever it was reliable enough, particularly for solutions with large periods in the range of weak nonintegrability.

[17] In principle the choice $(m k, m l)$ where $\mathrm{m}$ is some integer may either just reproduce the same periodic solution or represent a new one with $m$-times larger values of $P$ and $\phi_{P}$. Only the former possibility is realized numerically, so that all distinct periodic solutions are characterized by pairs of integers $(k, l)$ which do not have a common integer factor.

[18] The phase diagram in the $(B, C)$ plane with $\delta_{4}$ fixed which could be more useful for the discussions of particular physical examples has a rather complex structure which will be presented in the extended text [15].

[19] J. Villain and M. Gordon, J. Phys.C 13, 3117 (1980).

[20] A. Bjeliš and S. Barišić, Phys. Rev. Lett. 48, 684 (1982); S. Barišić and A. Bjeliš, in Electronic properties of organic materials with quasi-one-dimensional structure, edited by H. Kamimura (Riedel Publ. Comp., New York, Dordrecht, 1985), p.49. 


\section{FIGURES}

FIG. 1. Mean free energy vs $\phi_{0}^{\prime}$ for $\delta_{4}=\pi / 12, B=0.002, C=0.002[\mathrm{a}], B=0.008, C=0.006$ [b] and $B=0.02, C=0.02[\mathrm{c}]$ of periodic $(\triangle)$, quasiperiodic $(\bigcirc)$ and chaotic (solid $\diamond)$ solutions. The $(k, l)$ indices for the periodic solution with the lowest free energy (solid $\triangle)$ are $(3,4)$ in the

figures $[\mathrm{a}]$ and $[\mathrm{b}]$, and $(1,1)$ in the figure $[\mathrm{c}]$. The insets in figures [a] and [b] are enlarged neighborhoods of the free energy minima.

FIG. 2. The fundamental wave number $q_{0}$ vs $C$ and $\delta_{4}$ for $B=0.02$. The fractions at particular steps denote the ratios $2\left(\delta_{4}-q_{0}\right) / \pi=l /(4 k+3 l)$. In front of the dotted cross-section the rotational part of the phase space does not contain quasiperiodic solutions but only a single chaotic layer and isolated periodic solutions [see eq. (6)].

FIG. 3. Free energy of the absolutely stable periodic configurations vs $C$ and $\delta_{4}$ for $B=0.02$. The fractions and the dotted cross-section have the same meaning as in Fig.2. Note the reversed scales at the $\delta_{4}$ axes in Figs. 2 and 3. 
Fig. 1

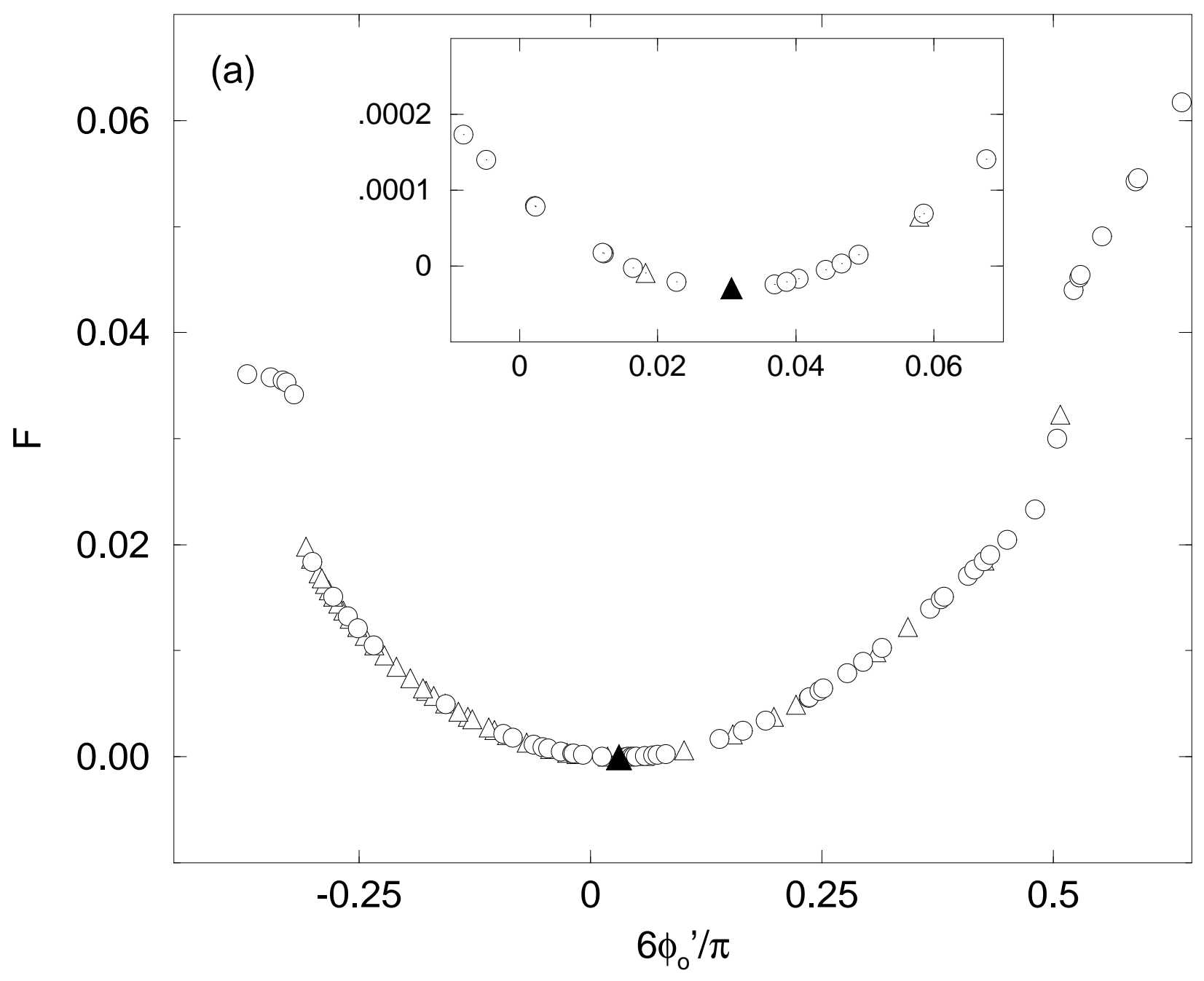


Fig. 1

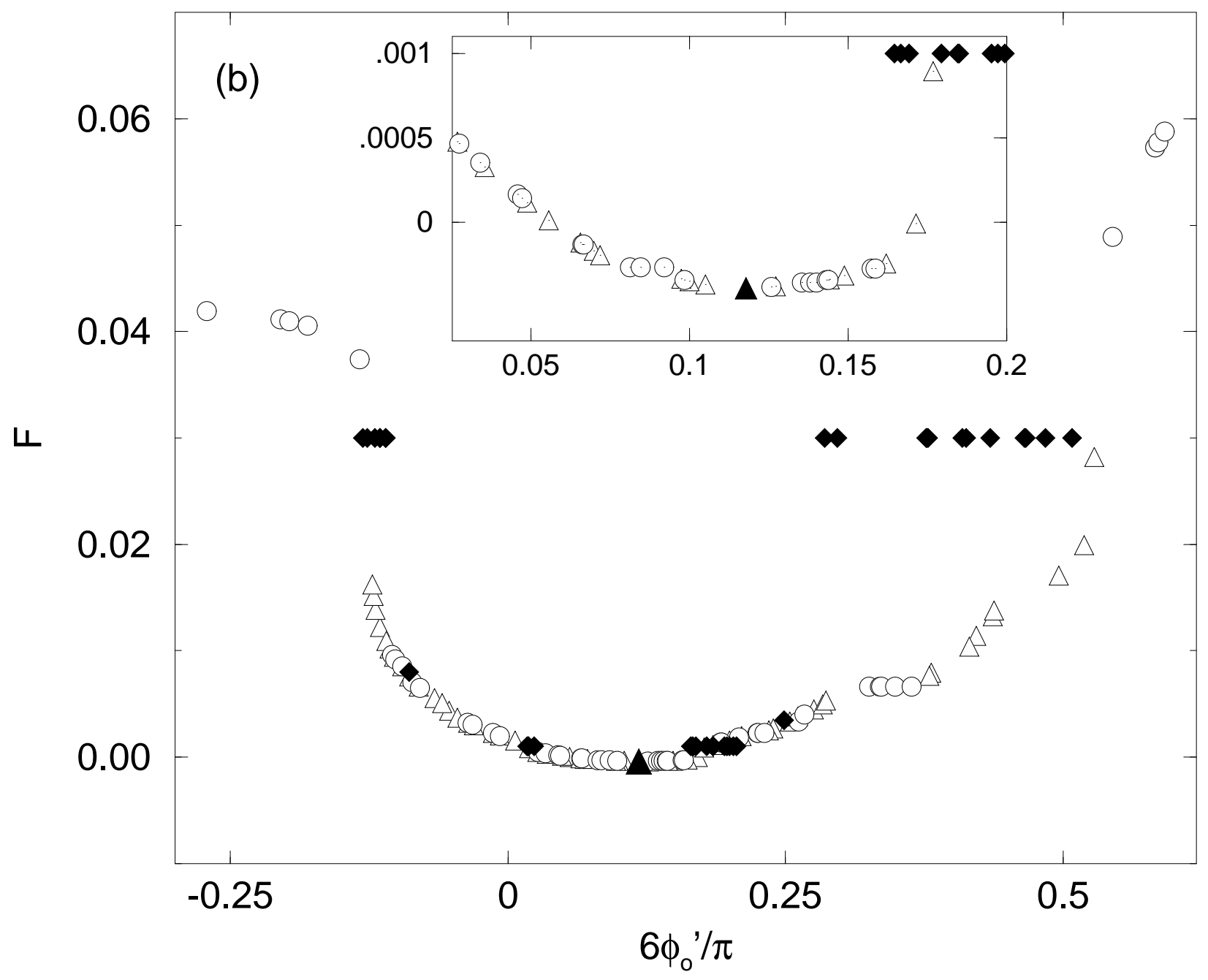


Fig. 1

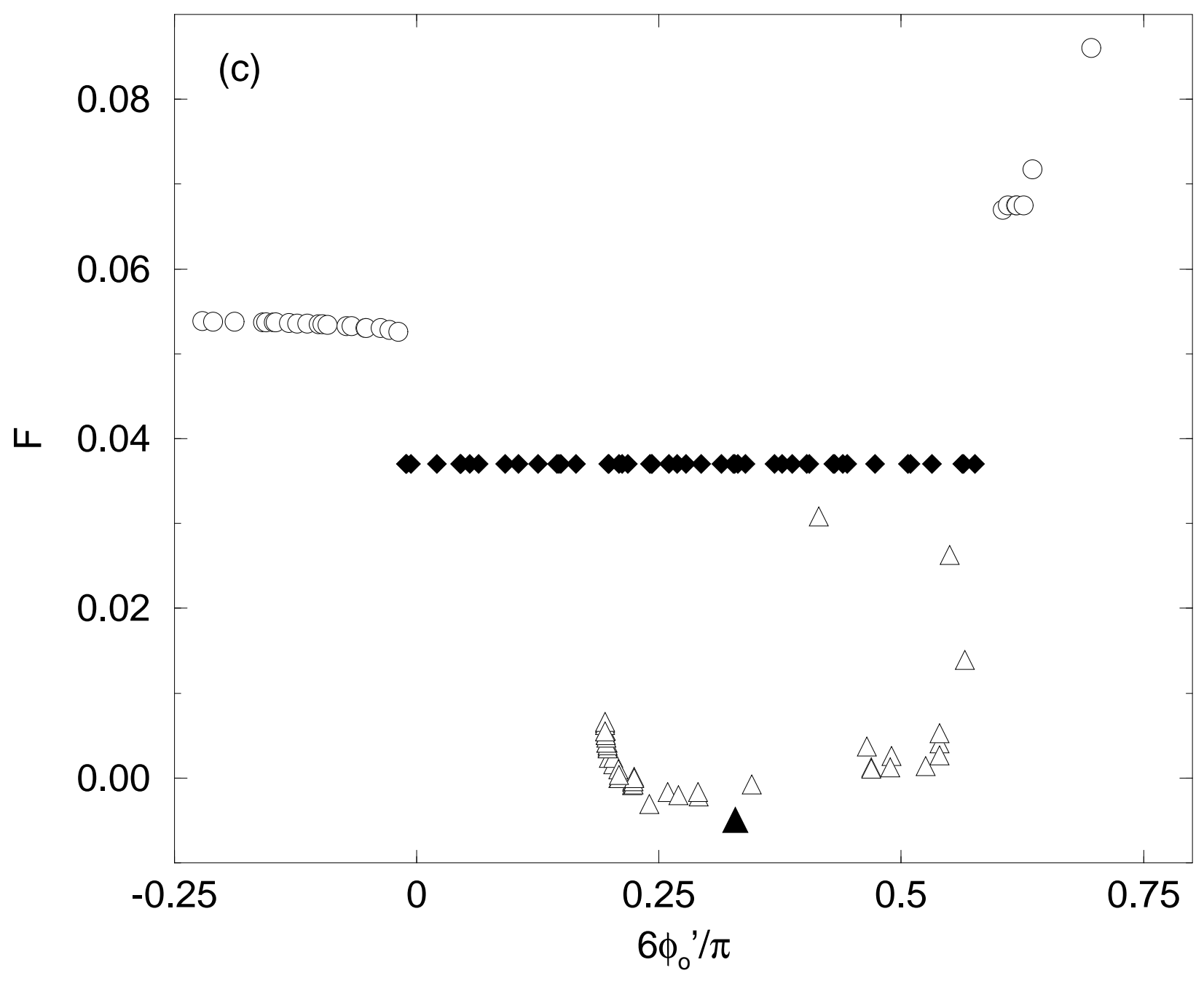


Fig. 2

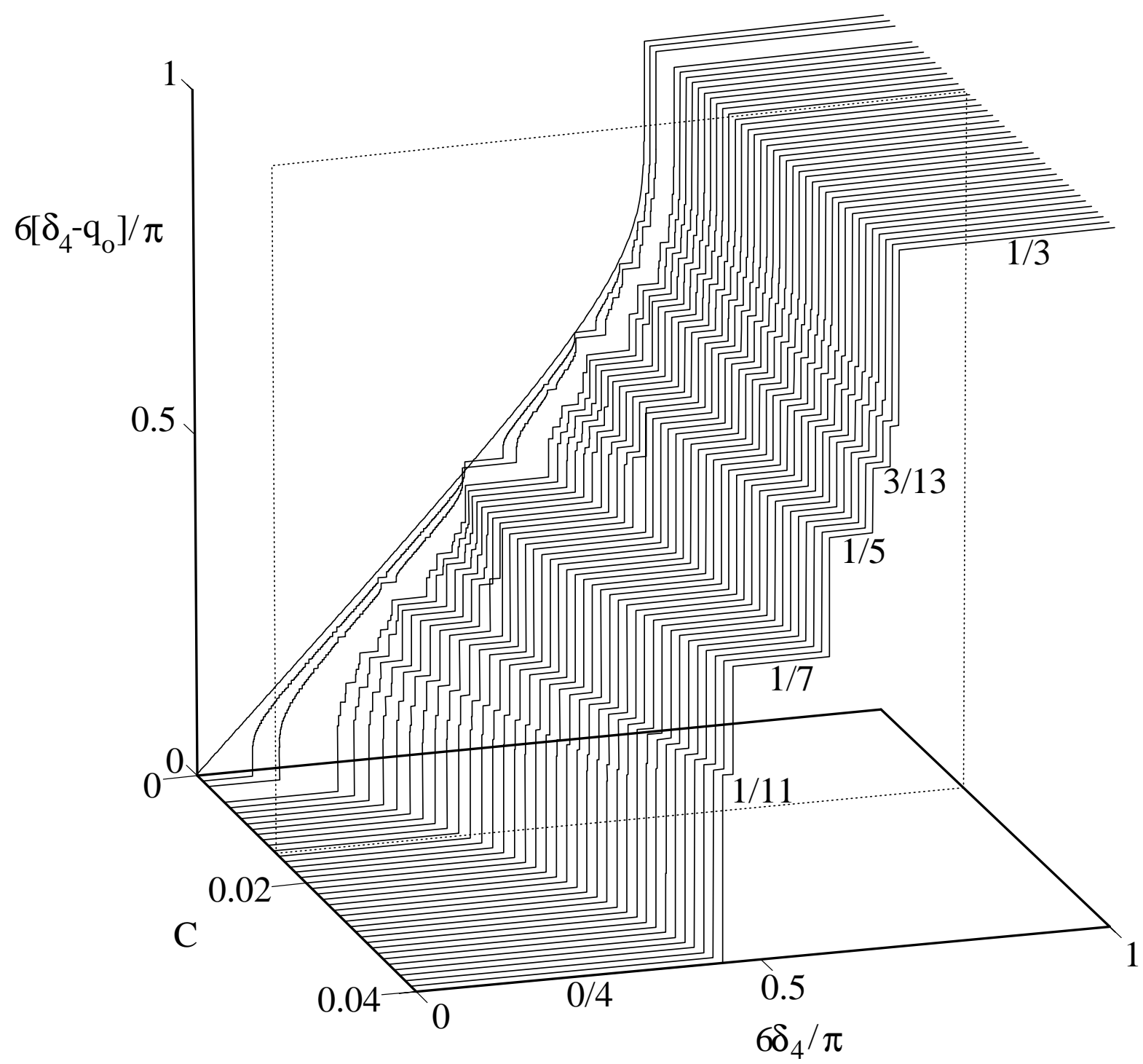


Fig. 3

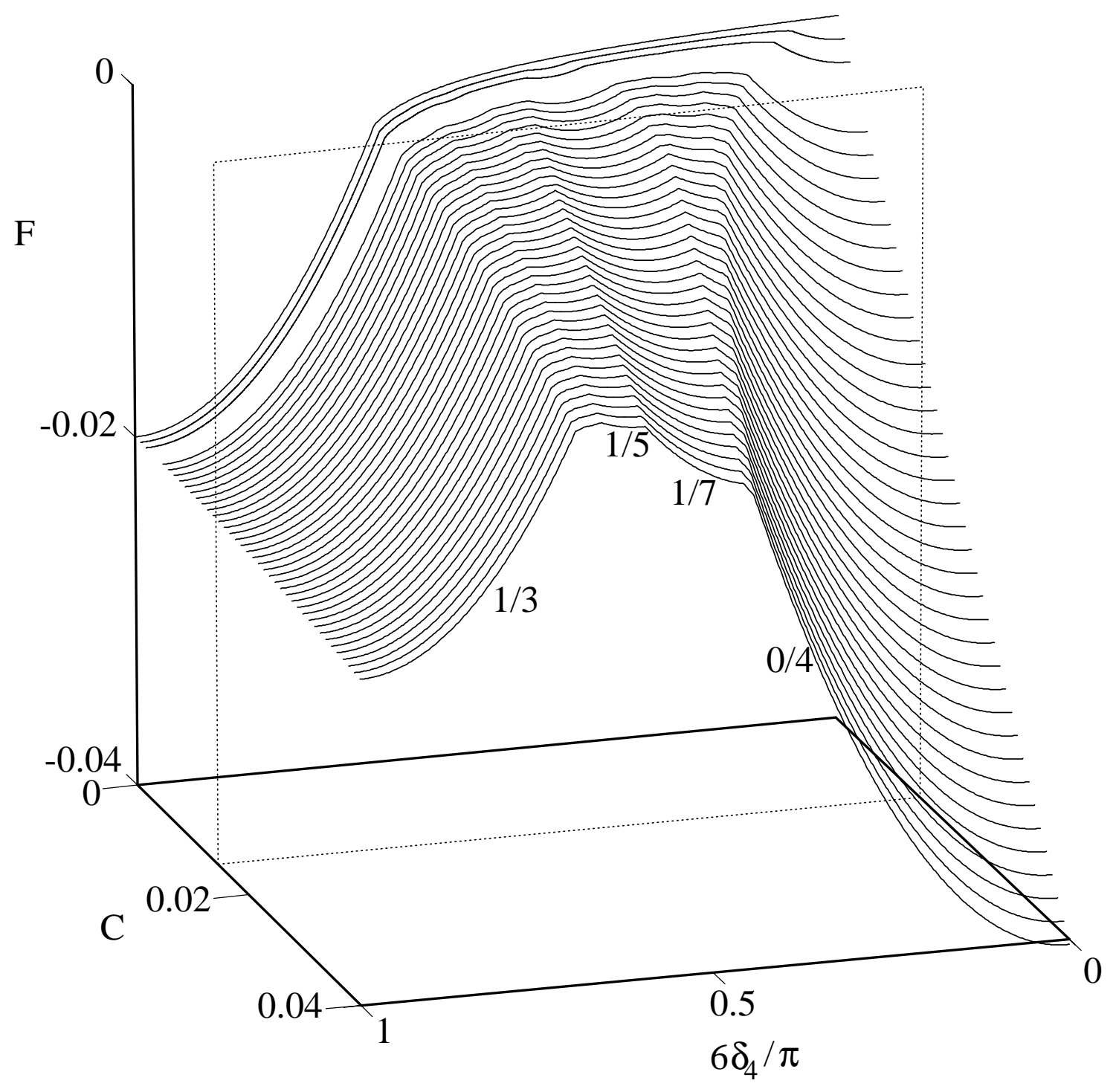

